\title{
Effect of Molecular Orientation on Photovoltaic Efficiency and Carrier Transport in a New Semiconducting Polymer
}

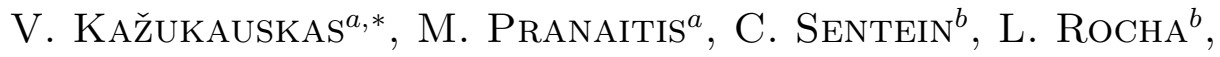 \\ P. Raimond ${ }^{b}$, I. Duyssens ${ }^{c}$, I. VAn Severen ${ }^{c}$, T. CleiJ ${ }^{d}$, \\ L. $\operatorname{LUTSEN}^{c}$ AND D. VANDERZANDE ${ }^{c, d}$ \\ ${ }^{a}$ Department of Semiconductor Physics \\ and Institute of Materials Science and Applied Research \\ Vilnius University, Saulètekio al. 9, bldg. 3, LT-10222 Vilnius, Lithuania \\ ${ }^{b}$ DRT-LITEN-DTS-LCS, bât.451, CEA Saclay, F-91191 Gif-sur-Yvette, France \\ ${ }^{c}$ IMEC-IMOMEC Division, Wetenschapspark 1, B-3590 Diepenbeek, Belgium \\ ${ }^{d}$ Hasselt University, Agoralaan 1, SBG/OS department \\ B-3590 Diepenbeek, Belgium
}

\begin{abstract}
New functionalized soluble poly ( $p$-phenylene vinylene) derivative bearing polar molecules was designed and synthesized in order to investigate effects of molecular orientation in polymer photovoltaic devices. The active polar molecule is the 4 -( $N$-butyl- $N$-2-hydroxyethyl)-1- nitro-benzene group. The grafting of the push-pull molecule with a donor/transmitter/acceptor structure, possessing a large ground state dipole moment, enables the molecular orientation by a dc electric field. An internal electric field stored in such system facilitates exciton dissociation and improves charge transport in single-layer devices. In our systems an increase in the external quantum efficiency by a factor of about 1.5 to 2 is estimated. The associated effects of orientation on the carrier injection and transport properties were evidenced.
\end{abstract}

PACS numbers: 73.50.-h, 81.40.Rs, 81.40.Tv, 73.61.Ph

\section{Introduction}

The storage of an internal electric field in polymer semiconductor devices is attractive for photovoltaic applications as it facilitates exciton dissociation and improves charge transport. The oriented diode-like molecules, the so-called pushpull molecules used in nonlinear optics [1], can be contained inside a polymer binder $[2,3]$. They can be oriented by a dc electric field, while heating the sample

*corresponding author; e-mail: vaidotas.kazukauskas@ff.vu.lt 
near the glass transition temperature. Oriented molecules induce a rectifying effect, behaving as a distributed homojunction within a single layer. The influence of orientation on charge injection, transport and trapping was confirmed in a copolymer of poly(methyl methacrylate) (PMMA) with Disperse Red 1 (DR1) $[2,3]$ and in the blends of poly(9-vinylcarbazole) (PVK) with 4-dibutylamino$4^{\prime}$-nitrostilbene (DBANS) [4]. The effect of orientation could be enhanced and stabilized in time if the polar molecules are not blended in a polymer binder, but are chemically grafted to the non-polar polymer chains.

\section{Samples and experiment}

We have investigated a new purposefully designed and synthesized functionalized soluble poly ( $p$-phenylene vinylene) (PPV) derivative bearing push-pull like molecules (NLO-PPV). The synthesis route will be described elsewhere. The active polar molecule is the 4-( $N$-butyl- $N$-2-hydroxyethyl)- 1-nitro-benzene group on the copolymer (Fig. 1a). It is a push-pull molecule with a donor/transmitter/acceptor structure, possessing a large ground state dipole moment which is necessary for the orientation. The polar molecule is attached onto a soluble PPV copolymer containing $50 \%$ of poly[2-methoxy-5-(3',7'-dimethyloctyloxy)]-1,4-phenylene vinylene (MDMO-PPV) units. This copolymer is used as an isotropic binder as it is one of the best conjugated polymers leading to high solar efficiency. $70 \mathrm{~nm}$ thick samples were spin-coated on the indium tin oxide(ITO) glass from a chlorobenzene solution and provided with semi-transparent $\mathrm{Al}$ electrodes.

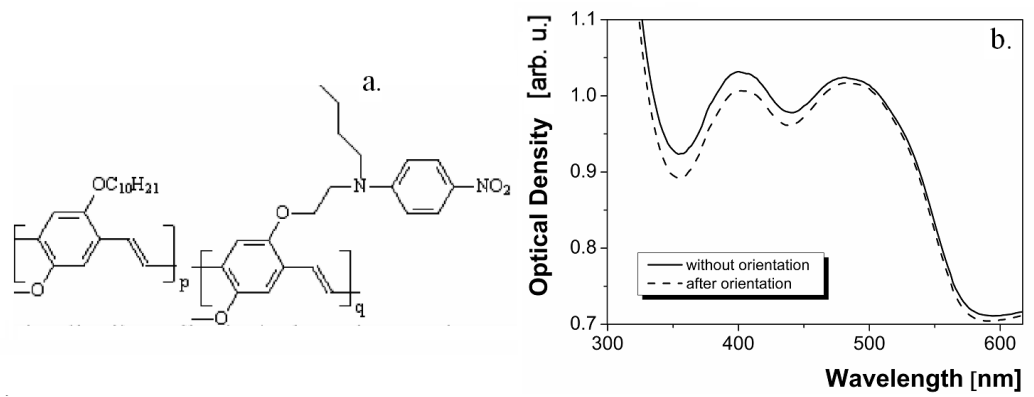

Fig. 1. (a) Chemical structure of the newly synthesized soluble NLO-PPV copolymer. (b) Absorption spectra of the device without orientation and after the orientation in forward direction.

The molecular orientation was performed in a dc field near the glass transition temperature. The reverse bias was used to create polarization field oriented in the forward direction and vice versa. The appearance of the built-in field was proven optically by the solid electric field induced second harmonic generation (SEFISHG) method. The effects of orientation on the carrier injection and transport properties were evidenced by the $I-V$ characteristics and mobility measurements. All changes were repeatable after several polarization-depolarization thermal cycles. 


\section{Results and discussion}

The orientation of polar molecules is evidenced by the absorption spectra (Fig. 1b). Their alignment perpendicularly to the sample surface causes the decrease of absorption. Typical $I-V$ dependencies with the bias applied to the $\mathrm{Al}$ electrodes are shown in Fig. 2a). Polarization with the positive voltage resulted in the growth of the current, meanwhile polarization with the negative voltage led at low biases to its decrease.
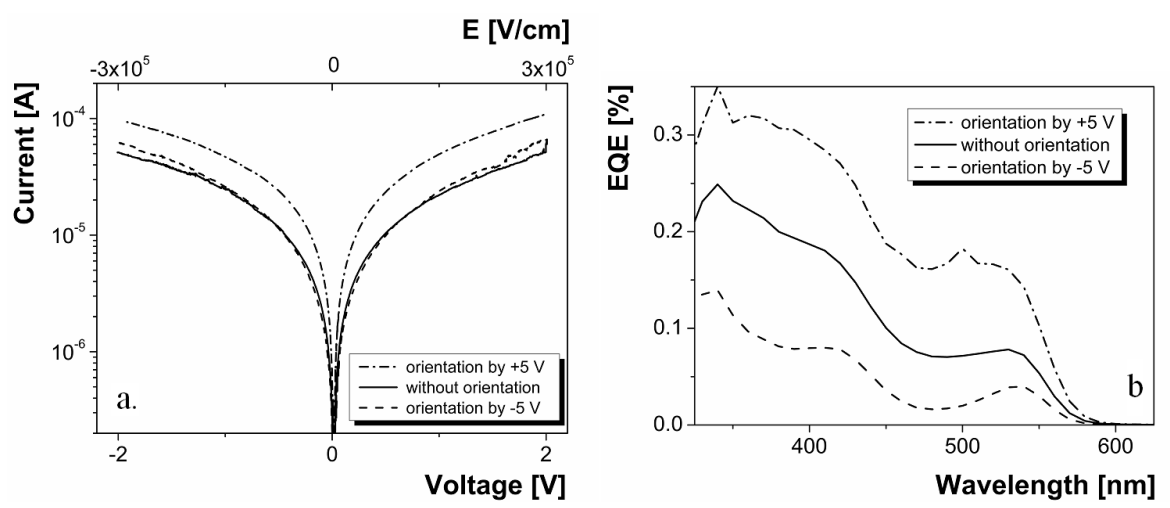

Fig. 2. Effect of the molecular orientation on the mobility dependencies vs. applied electric field strength. (a) - $I-V$ characteristics, and (b) - external quantum efficiency spectra.

An integral increase of the External Quantum Efficiency (EQE) by a factor of $1.5 \div 2$ was evaluated over the visible region (Fig. $2 \mathrm{~b}$ ) upon orientation. Since the EQE increases over the whole spectrum, this is not due to a purely optical modification, as, e.g., absorption (Fig. 1b). These observations are consistent with the $I-V$ and mobility data, confirming the role of carrier transport. The conditions for the carriers to move are improved after the orientation in forward direction and carrier transport is aggravated after the reverse orientation. In the tested devices the EQE remains lower as compared to the solar cells on the MDMOPPV. However, the test devices were not yet optimised in terms of the absorption and charge collection efficiency.

Carrier mobility dependencies on the orientation and applied electric field differ significantly at low fields (Fig. 3a). This is because only a part of molecules are oriented, and the strength of the built-in polarization field is limited. Characteristically, dependence of mobility on the orientation at lower fields up to $(1 \div 1.5) \times 10^{5} \mathrm{~V} / \mathrm{cm}$ qualitatively coincides with the behaviour of the I-V curves, evidencing the important role of mobility. Nevertheless differences in current at higher fields in Fig. 2a) cannot be attributed to the mobility, which becomes independent on the polarization. Probably, modification of contact or surface effects cannot be excluded in this case. 

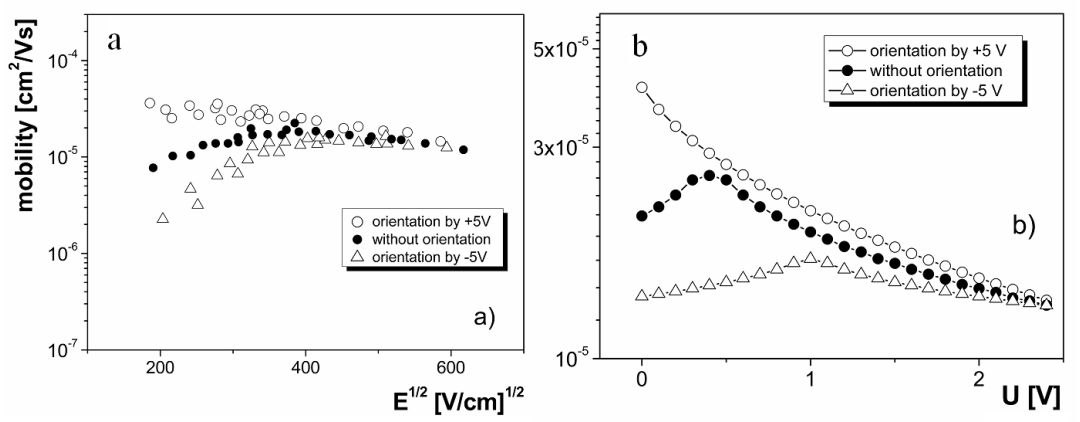

Fig. 3. Effect of the molecular orientation on the mobility dependencies vs. applied electric field strength. (a) Experimental data, (b) modelling results.

The unusual mobility maximum appears in Fig. 3. To our knowledge, such behavior was observed for the first time. We have modelled it (Fig. 3b) using the Gaussian disorder model (GDM) [5] and taking into account the superposition of three electric fields present in the system: $\boldsymbol{E}=\boldsymbol{E}_{\text {cont }}+\boldsymbol{E}_{\text {orient }}+\boldsymbol{E}_{\text {exit }}$. Here $\boldsymbol{E}_{\text {cont }}$ is the built-in contact field given by the different work functions of Al and ITO, $\boldsymbol{E}_{\text {orient }}$ is the orientation-induced internal field and $\boldsymbol{E}_{\text {ext }}$ is the applied external field. The observed maximum can appear in a system in which mobility diminishes with increasing resulting electric field strength. This so called "negative" mobility dependence is characteristic of the spatially inhomogeneous systems. By time of flight similar effect was observed many times in different disordered materials as, e.g., molecularly doped polymers, molecular glasses, etc. In polymers, the negative mobility behavior was first evidenced in P3HT in [6] and P3OT in [7].

\section{Acknowledgments}

We acknowledge partial financial support by the European Commission through the FP6 project MOLYCELL, contract No. SES6- CT-2003-502783.

\section{References}

[1] Ch. Bosshard, K. Sutter, Ph. Prêtre, J. Hulliger, M. Flörsheimer, P. Kaatz, P. Günter, Organic Nonlinear Optical Materials 1, Gordon and Breach, New York 1995.

[2] C. Sentein, C. Fiorini, A. Lorin, J.-M. Nunzi, Adv. Mater. 9, 809 (1997).

[3] C. Sentein, C. Fiorini, A. Lorin, J.-M. Nunzi, Synthetic Metals 91, 81 (1997).

[4] V. Kažukauskas, V. Čyras, M. Pranaitis, A. Apostoluk, L. Rocha, L. Sicot, P. Raimond, C. Sentein, Org. Electron. 8, 21 (2007).

[5] H. Baessler, Phys. Status Solidi B 175, 15 (1993).

[6] A.J. Mozer, N.S. Sariciftci, A. Pivrikas, R. Österbacka, G. Juška, L. Brassat, H. Bässler, Phys. Rev. B 71, 035214 (2005).

[7] V. Kažukauskas, M. Pranaitis, L. Sicot, F. Kajzar, Mol. Cryst. Liq. Cryst. 447, 459 (2006). 